\title{
A Mathematical Model to Analyze Data on Coronavirus Cases
}

\author{
Santanu Basu \\ Sparkle Optics Corporation \\ Menlo Park, CA \\ sb201@sparkleoptics.com
}

\begin{abstract}
A mathematical model is presented which is designed to be applicable to Coronavirus case data from any country and which can serve as a tool to carry out predictive analysis and sensitivity analysis with the goal of minimizing virus related deaths. The model has been successfully applied to Covid-19 case data in five countries - China, South Korea, Iran, Italy and the USA. The model is versatile and can be applied to other countries and regions as well.
\end{abstract}

Key words: Coronavirus, Covid 19, modelling, mathematical, infectious disease, data analysis

\section{INTRODUCTION}

Mathematical modelling of spreading of infectious diseases is a well-established field [1,2]. Many infectious diseases such as Hepatitis C, HIV and Lyme disease have been extensively studied using mathematical models. There have been a surge of recent efforts in modelling Coronavirus spreading as reported in the literature [3,4]. In this paper we present a mathematical model which analyzes Covid-19 case data (daily values of number of cases, deaths and recovered) from five countries with very different case outcomes. The model fits the Covid-19 case data for these countries very well. The model also enables the reader to make near term predictions and sensitivity analysis for any country.

The Covid-19 virus has spread rapidly through many countries in the world in the first three months of 2020 causing widespread deaths. The same virus with the same infectious and fatality properties however had different number of casualties in different countries. It is important to have a mathematical model that can explain the case data from various countries and that uses parameters which can be measured and which can be used to do sensitivity and what if analyses. This is the motivation behind the model presented in this paper.

\section{METHODOLOGY}

In this section we study the propagation of a new infectious disease through an isolated country's population. With some simple modifications, the procedure can be applied to recurring outbreak of the same virus and also to the normal state in which there is population movement among countries.

Let $n$ be the number of days since the onset of virus infection.

Figure 1 is a schematic of our model. 


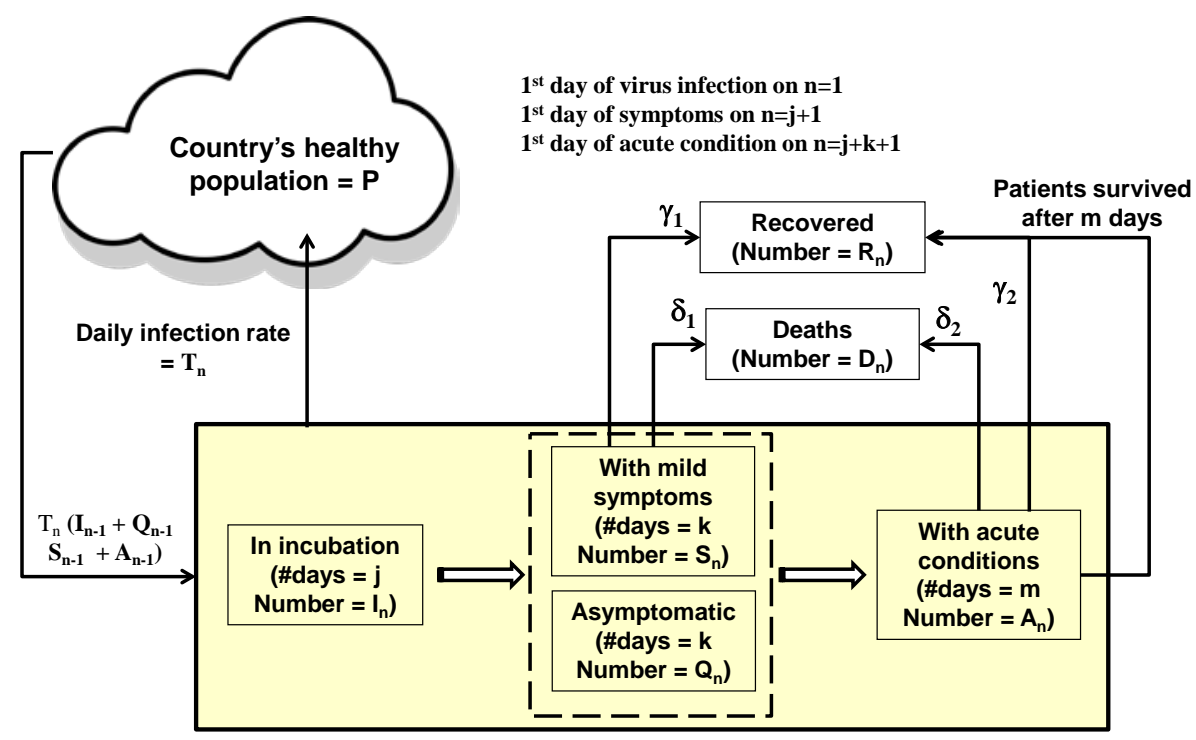

Snapshot on day $\mathbf{n}$ since virus outbreak

Symbols: daily recovery rate $=\gamma$, daily death rate $=\delta$, daily testing rate $=\beta$

Figure 1. Schematic of the mathematical model for the virus infected cases

The entire population of virus infected people is classified in six categories:

I - people in whom the virus is in incubation

$\mathrm{Q}$ - people who are infected with the virus but who do not exhibit any symptoms

$\mathrm{S}$ - patients who exhibit mild symptoms of the viral infection

A - patients who are in acute condition

$\mathrm{D}$ - people who have died as a result of viral infection

$\mathrm{R}$ - people who have recovered from the viral infection

On any day n, the total number of people who have been infected with the virus is $\mathrm{N}_{\mathrm{n}}$, where

$\mathrm{N}_{\mathrm{n}}=\mathrm{I}_{\mathrm{n}}+\mathrm{Q}_{\mathrm{n}}+\mathrm{S}_{\mathrm{n}}+\mathrm{A}_{\mathrm{n}}+\mathrm{D}_{\mathrm{n}}+\mathrm{R}_{\mathrm{n}}$

We denote the incubation period for the virus in number of days by $\mathrm{j}$. The model assumes each infected person spends $\mathrm{j}$ days in incubation. We assume that after the incubation period of $\mathrm{j}$ days, each infected person either is asymptomatic but carries the virus for another k number of days, or shows mild symptoms of the disease for k number of days. For each patient during each day in this mild symptom phase, the probabilities of recovery and death are denoted by $\gamma_{1}$ and $\delta_{1}$ per day. At the end of $k$ number of days, the patients who have not recovered or died are assumed to enter the acute condition phase as shown in figure 1 . We assume that the acute condition period lasts for $\mathrm{m}$ number of days. For each patient during each day in the acute condition phase, the probabilities of recovery and death are denoted by $\gamma_{2}$ and $\delta_{2}$ per day. The patients who survive $m$ days in acute condition are assumed to be fully recovered. 
Let $I_{n}$ be the total population of infected people who are still in the incubation period on day $n$. Let $\mathrm{I}_{\mathrm{n}}{ }^{\mathrm{i}}$ be the population of those infected people in the incubation period on day $n$ who have been infected for i number of days (i ranges from 1 to $\mathrm{j}$ ).

$$
\mathrm{I}_{\mathrm{n}}=\mathrm{I}_{\mathrm{n}}{ }^{1}+\mathrm{I}_{\mathrm{n}}{ }^{2}+\ldots . .+\mathrm{I}_{\mathrm{n}}{ }^{\mathrm{j}}=\sum^{\mathrm{i}} \mathrm{I}_{\mathrm{n}}{ }^{\mathrm{i}}
$$

Let $S_{n}$ be the total population of infected people who are showing mild symptoms of the disease on day $n$. Let $S_{n}{ }^{i}$ be the population on day $n$ of those infected patients who have been showing mild symptoms for i number of days (i ranges from 1 to $\mathrm{k}$ ).

$$
\mathrm{S}_{\mathrm{n}}=\mathrm{S}_{\mathrm{n}}{ }^{1}+\mathrm{S}_{\mathrm{n}}{ }^{2}+\ldots . .+\mathrm{S}_{\mathrm{n}}{ }^{\mathrm{k}}=\sum^{\mathrm{i}} \mathrm{S}_{\mathrm{n}}{ }^{\mathrm{i}}
$$

Let $\mathrm{Q}_{\mathrm{n}}$ be the total population of infected people who have gone through the incubation period and in whom the virus still lives for k number of days, but who are asymptomatic of the disease. Let $\mathrm{Q}_{\mathrm{n}}{ }^{\mathrm{i}}$ be the population on day n of those asymptomatic people who have been carrying the virus for $\mathrm{j}+\mathrm{i}$ number of days ( $\mathrm{i}$ ranges from 1 to $\mathrm{k}$ ).

$$
\mathrm{Q}_{\mathrm{n}}=\mathrm{Q}_{\mathrm{n}}{ }^{1}+\mathrm{Q}_{\mathrm{n}}{ }^{2}+\ldots .+\mathrm{Q}_{\mathrm{n}}{ }^{\mathrm{k}}=\sum^{\mathrm{i}} \mathrm{Q}_{\mathrm{n}}{ }^{\mathrm{i}}
$$

Let $A_{n}$ be the total population of patients who are in acute condition on day n. Let $A_{n}{ }^{i}$ be the population on day $n$ of those infected patients who have been in acute condition for i number of days (i ranges from 1 to $\mathrm{m}$ ).

$$
A_{n}=A_{n}{ }^{1}+A_{n}{ }^{2}+\ldots . .+A_{n}{ }^{m}=\sum^{i} A_{n}{ }^{i}
$$

The total number of people who are currently infected on day $n$ and who are able to transmit the virus is $\left(I_{n}+S_{n}+Q_{n}+A_{n}\right)$. Out of this population, only $\left(S_{n}+A_{n}\right)$ number of people show outward signs of the disease. Ironically in the beginning of the outbreak, when $\left(S_{n}+A_{n}\right)$ is small, the problem is sometimes underestimated without realizing that the disease is spreading exponentially everyday by the entire infected population $\left(\mathrm{I}_{n}+\mathrm{S}_{\mathrm{n}}+\mathrm{Q}_{\mathrm{n}}+\mathrm{A}_{\mathrm{n}}\right)$. The time to take swift and decisive preventive measures is when $\left(S_{n}+A_{n}\right)$ is small.

We denote the rate of transmission of the virus from an infected person to a healthy person per day by $\mathrm{T}_{\mathrm{n}}$. Let $\mathrm{N}_{0}$ be the number of infected people on the first day. For example, the numbers of infected people on the first two days of the outbreak are:

$$
\begin{aligned}
& \mathrm{I}_{1}=\mathrm{N}_{0} \\
& \mathrm{I}_{2}=\mathrm{N}_{0}\left(1+\mathrm{T}_{1}\right)
\end{aligned}
$$

In general, the number of newly infected people on day $n$ is given by

$$
\mathrm{I}_{\mathrm{n}}{ }^{1}=\mathrm{T}_{\mathrm{n}-1}\left(\mathrm{I}_{\mathrm{n}-1}+\mathrm{S}_{\mathrm{n}-1}+\mathrm{Q}_{\mathrm{n}-1}+\mathrm{A}_{\mathrm{n}-1}\right)
$$

A fraction, $\mathrm{f}_{\mathrm{a}}$, of the newly infected people is asymptomatic of the disease. An estimate of $\mathrm{f}_{\mathrm{a}}=$ $30 \%$ has been given in reference [9], which has been used in this model calculations. The number of new patients with mild symptoms on day $n$ is given by

$$
\mathrm{S}_{\mathrm{n}}{ }^{1}=\left(1-\mathrm{f}_{\mathrm{a}}\right) \mathrm{I}_{\mathrm{n}-1}{ }^{\mathrm{j}}
$$

Similarly the number of new asymptomatic patients on day $n$ is given by

$$
\mathrm{Q}_{\mathrm{n}}{ }^{1}=\mathrm{f}_{\mathrm{a}} \mathrm{I}_{\mathrm{n}-1}{ }^{\mathrm{j}}
$$

The number of new patients with acute condition on day $n$ is given by 
$\mathrm{A}_{\mathrm{n}}{ }^{1}=\left(1-\gamma_{1}-\delta_{1}\right) \mathrm{S}_{\mathrm{n}-1}^{\mathrm{k}}$

The numbers of patients who recover and die from mild symptoms on day $n$ are given by $\gamma_{1} S_{n}$ and $\delta_{1} \mathrm{~S}_{\mathrm{n}}$ respectively. Similarly the numbers of patients who recover and die from acute conditions on day $n$ are given by $\gamma_{2} A_{n}$ and $\delta_{2} A_{n}$.

$$
\begin{aligned}
& \mathrm{S}_{\mathrm{n}}{ }^{\mathrm{i}+1}=\left(1-\gamma_{1}-\delta_{1}\right) \mathrm{S}_{\mathrm{n}-1}{ }^{\mathrm{i}} \text { for } 0 \leq \mathrm{i} \leq \mathrm{k}-1, \mathrm{n}>1 \\
& \mathrm{Q}_{\mathrm{n}}{ }^{\mathrm{i}+1}=\mathrm{Q}_{\mathrm{n}-1}{ }^{\mathrm{i}} \text { for } 0 \leq \mathrm{i} \leq \mathrm{k}-1, \mathrm{n}>1 \\
& \mathrm{~A}_{\mathrm{n}}{ }^{\mathrm{i}+1}=\left(1-\gamma_{2}-\delta_{2}\right) \mathrm{A}_{\mathrm{n}-1}{ }^{\mathrm{i}} \text { for } 0 \leq \mathrm{i} \leq \mathrm{k}-1, \mathrm{n}>1
\end{aligned}
$$

The number of deaths caused by the virus on day $n$ is given by

$$
\mathrm{D}_{\mathrm{n}}=\delta_{1} \mathrm{~S}_{\mathrm{n}}+\delta_{2} \mathrm{~A}_{\mathrm{n}}
$$

The number of patients who recover from the disease on day $\mathrm{n}$ is given by

$$
R_{n}=\gamma_{1} S_{n}+\gamma_{2} A_{n}+\left(1-\delta_{2}\right) A_{n}{ }^{m}
$$

Before the first person showing symptoms of the disease caused by the new virus and shortly thereafter up to day $\mathrm{n}_{1}$, the transmission rate $\mathrm{T}_{\mathrm{n}}$ is assumed to be constant at an initial value $\mathrm{T}_{0}$ :

$$
\mathrm{T}_{\mathrm{n}}=\mathrm{T}_{0} \text { for } 1 \leq \mathrm{n} \leq \mathrm{n}_{1}
$$

Typically the authorities take steps to prevent the spreading of the disease such as patient isolation, testing, contact tracing and social distancing. As a result $\mathrm{T}_{\mathrm{n}}$ decreases with time. The model is capable of including actual time dependent transmission data, however at this time such data for the Coronavirus case is not available. A reasonable approximation is exponential decay of the rate of transmission $\mathrm{T}_{\mathrm{n}}$ from infected persons to the general population. Let $\mathrm{G}$ be the number of days it takes for $T_{n}$ to decrease by $63 \%$ of $T_{0}$, and let $T_{f}$ be the long term steady state value of $T_{n}$.

$$
\mathrm{T}_{\mathrm{n}}=\mathrm{T}_{\mathrm{f}}+\left(\mathrm{T}_{0}-\mathrm{T}_{\mathrm{f}}\right) \exp \left(-\left(\mathrm{n}-\mathrm{n}_{1}\right) / \mathrm{G}\right) \text { for } \mathrm{n}_{1}<\mathrm{n}
$$

Three parameters $n_{1}, G$ and $T_{f}$ were found to significantly affect the number of cases from the virus. The data analyses carried out in this paper clearly showed that the actual case data for countries with smaller number of cases could be fit well with smaller $G$ and $n_{1}$. In this paper, we will limit the analysis to the first outbreak of the virus. It may also happen that social distancing measures are lifted due to economic necessity before the virus is completely extinguished within the population, which would cause $T_{n}$ to rise again and a possible recurrence. The recurrence can be modelled in future using the methodology given in this paper.

Let $\sigma_{\mathrm{n}}$ per day per person be the rate of testing of the entire population, $\mathrm{P}$ of the country including the infected people without symptoms $\left(\mathrm{I}_{n}+\mathrm{Q}_{\mathrm{n}}\right)$ on any day $\mathrm{n}$. Let $\beta_{\mathrm{n}}$ per day per person be the testing rate of patients with symptoms. The active number of infected cases which are confirmed by testing and reported can be expressed as:

$$
B_{n}=\sigma_{n}\left(I_{n}+Q_{n}\right)+\beta_{n}\left(S_{n}+A_{n}\right)
$$

Total cumulative number of reported cases, $C_{n}$ is given by

$\mathrm{C}_{\mathrm{n}}=\mathrm{B}_{\mathrm{n}}+\sum^{\mathrm{i}} \beta_{\mathrm{i}}\left(\mathrm{D}_{\mathrm{i}}+\mathrm{R}_{\mathrm{i}}\right)$ for $1 \leq \mathrm{i} \leq \mathrm{n}$

In the model, the testing rates $\sigma_{\mathrm{n}}$ and $\beta_{\mathrm{n}}$ are considered time dependent as follows: 


$$
\begin{aligned}
& \beta_{\mathrm{n}}=0 \text { for } 1 \leq \mathrm{n} \leq \mathrm{j} \\
& \beta_{\mathrm{n}}=\beta_{0} \text { for } \mathrm{j}+1 \leq \mathrm{n} \leq \mathrm{n}_{2} \\
& \beta_{\mathrm{n}}=\beta_{0}+\left(\left(1-\beta_{0}\right)\left(\mathrm{n}-\mathrm{n}_{2}\right) / \mathrm{n}_{\beta}\right) \text { for } \mathrm{n}_{2} \leq \mathrm{n} \leq \mathrm{n}_{2}+\mathrm{n}_{\beta} \\
& \beta_{\mathrm{n}}=1 \text { for } \mathrm{n}>\mathrm{n}_{2}+\mathrm{n}_{\beta} \\
& \text { and, } \sigma_{\mathrm{n}}=0 \text { for } 1 \leq \mathrm{n} \leq \mathrm{n}_{2}+\mathrm{n}_{\beta} \\
& \sigma_{\mathrm{n}}=\sigma_{\mathrm{f}}\left(\left(\mathrm{n}-\left(\mathrm{n}_{2}+\mathrm{n}_{\beta}\right)\right) / H\right) \text { for } \mathrm{n}_{2}+\mathrm{n}_{\beta} \leq \mathrm{n} \leq \mathrm{n}_{2}+\mathrm{n}_{\beta}+H \\
& \sigma_{\mathrm{n}}=\sigma_{\mathrm{f}} \text { for } \mathrm{n}>\mathrm{n}_{2}+\mathrm{n}_{\beta}+H
\end{aligned}
$$

The rationale for choosing different testing rates is as follows. During the first $\mathrm{j}$ number of incubation days of the outbreak there is no testing and $\beta_{\mathrm{n}}=0$. During the first outbreak of the virus, it takes several days to identify the new virus and to develop an initial test, during which time, the testing rate of the patients showing symptoms is low, denoted by $\beta_{0}$. Let $n_{\beta}$ be the number of days to ramp up the testing of the patients with symptoms to $100 \%$ final testing rate. The testing rate $\sigma_{\mathrm{n}}$ for the general population including those who are infected and not showing symptoms is assumed to be zero until $n=n_{2}+n_{\beta}$ during which time testing rate is being ramped up for those showing symptoms. Let $\mathrm{H}$ be number of days it takes to build up the testing capability to test the general population at a final rate of $\sigma_{\mathrm{f}}$. This recognizes that large scale production of testing supplies and development of infrastructure and resources for administering of tests in large numbers take time.

It is to be noted that there is daily variation and probability distribution function for each of these model parameters. The time dependent data on these parameters for the new Coronavirus in each country are not available yet. When the values of these parameters become available on a later date, these can be inserted in the model. For near real time analysis of the case data, we make realistic initial assumptions as described in detail in this section and then numerically iterate on them to fit the published data on Covid-19 infected cases for each country.

\section{Model parameters}

We test this model to explain the number of Covid-19 cases in a small number of countries at first China, South Korea, Iran, Italy and USA. We collected the data on number of cases, deaths and number of recovered people from reliable sources [5-8] for the period January $1^{\text {st }}, 2020$ to March $31^{\text {st }}, 2020$. The actual data used in this paper is included in table I in the appendix.

There are seventeen variable parameters in the model description. These are $\mathrm{j}, \mathrm{k}, \mathrm{m}, \mathrm{N}_{\mathrm{o}}, \mathrm{T}_{\mathrm{o}}, \mathrm{T}_{\mathrm{f}}$, $\mathrm{n}_{1}, \mathrm{G}, \beta_{0}, \mathrm{n}_{2}, \mathrm{n}_{\beta}, \gamma_{1}, \gamma_{2}, \delta_{1}$ and $\delta_{2}, \sigma_{\mathrm{f}}$ and $H$. We start with reasonable assumptions for each parameter and then use the published data on total number of cases, total number of deaths and total number of recovered patients for each country to iteratively optimize the parameter values using the mathematical model. We also make sure that the values for the parameter are reasonable. For example, for $\mathrm{j}$, $\mathrm{k}$ and $\mathrm{m}$, we make use of the information presented on the WHO website [5], which states "Most estimates of the incubation period for COVID-19 range from 1-14 days, most commonly around five days. These estimates will be updated as more data become available." Another such source of information is a published study done at the World Health Organization, "Using available preliminary data, the median time from onset to clinical recovery for mild cases is 
approximately 2 weeks and is 3-6 weeks for patients with severe or critical disease. Preliminary data suggests that the time period from onset to the development of severe disease, including hypoxia, is 1 week. Among patients who have died, the time from symptom onset to outcome ranges from 2-8 weeks. "[6].

We carry out multivariate optimization to fit the data for each country between Jan 1 and Mar 31 of 2020. Starting with reasonable initial conditions in the model, iterations are made in the algorithm until the residual errors in fitting the published data during Jan 1 and Mar 31, 2020 for each country are minimized. At that point the final values of all parameters for this predictive model for each country become available. This can be considered as the training set. Once the optimization is completed, the country-specific parameters are determined and the model becomes powerful to yield information such as (1) predicted number of future cases in a country or (2) sensitivity analysis such as the effect on number of casualties on days to implement social distancing. The model can also be used to quantify if there is a recurrence of the disease once social distancing measures are lifted. We discuss the results in the next section.

\section{RESULTS AND DISCUSSION}

We used the data on number of cases, deaths and number of recovered people from reliable sources [5-8] for the period January $1^{\text {st }}, 2020$ to March $31^{\text {st }}$, 2020. The actual data used in this paper is included in table I in the appendix. The results are shown for China, South Korea, Iran, Italy and USA in figures 2-6. The same model fits the data for five countries in three continents remarkably well as shown in the figures. As stated before, we have done multivariable optimization and iterated on seventeen parameters to obtain the best fit between the model prediction and the actual data for each country.

Figure 2 shows the comparison of the actual data and the model predictions for China in five categories: (a) total cases, (b) total deaths, (c) total recovered, (d) daily new cases and (e) daily deaths. In the literature [5,6,8], the daily case data for China became available since Jan 22, 2020. Before that time, the data has been scarce. On Dec 19, 40 cases were reported in China, 59 cases were reported on Jan 9, 200 cases were reported on Jan 20 and 571 cases were reported on Jan 22. The $1^{\text {st }}$ death was reported on Jan 11, a total of 2 deaths were reported on Jan 17 which increased to 3 on Jan 20 and to 17 on Jan 22.

We used the mathematical model to best fit the case data for China between Jan 1 and Mar 31, 2020 and to obtain the values for parameters used in the model for China. For example, the best fit for the data with minimum least squares error was obtained by using initial number of infected people, $\mathrm{N}_{0}=10$, initial transmission factor $\mathrm{T}_{0}=0.54$, Jan 15 start date for transmission factor decrease and a transmission decay rate of $1 / G$, where $G=6$ days. The spike in the actual daily case on Feb 12 could not be fitted with model, as it was a one-time event in the actual data set. It should also be mentioned that the case data from China was revised on April $17^{\text {th }}$ which is outside the data set used in this paper. 


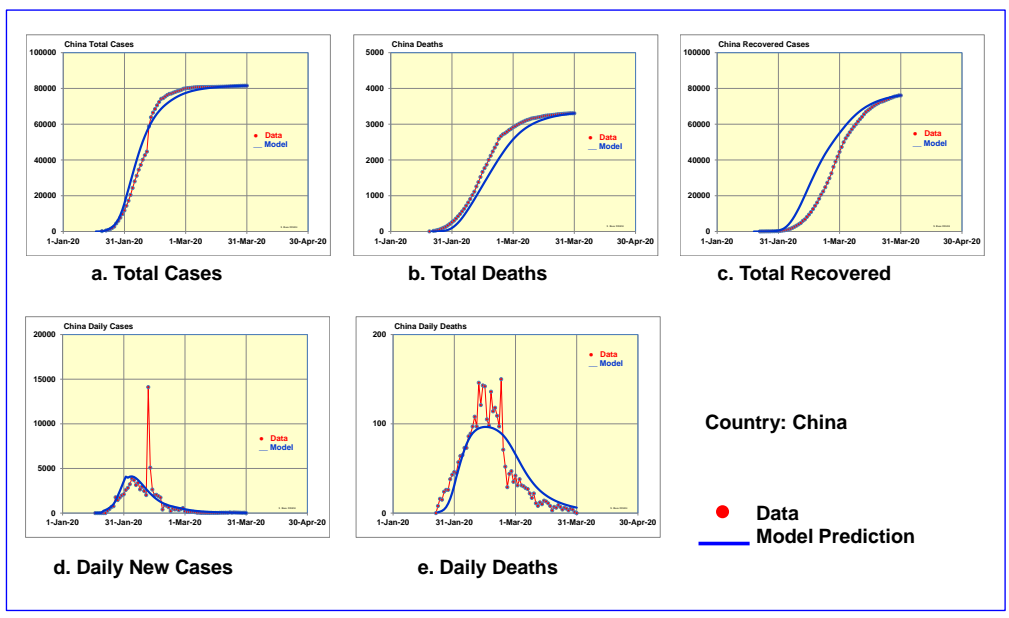

Figure 2. Data and model predictions for Covid-19 cases in China

Figure 3 shows the comparison of the actual data and the model predictions for South Korea. The daily case data for South Korea has been available in literature $[5,6,8]$ since Feb 15 . There were 28 total cases on Feb 15 and the first Covid-19 death was on Feb 20. The model fitted the South Korea case data with minimum error with $\mathrm{N}_{0}=3, \mathrm{~T}_{0}=0.47$, Feb 12 start date for transmission factor decrease and a transmission decay rate of $1 / G$, where $G=6$ days. Swift action to reduce the transmission factor when the infected population was small is what we believe to be the primary reason for very low Covid-19 deaths in South Korea and which is supported by the model.

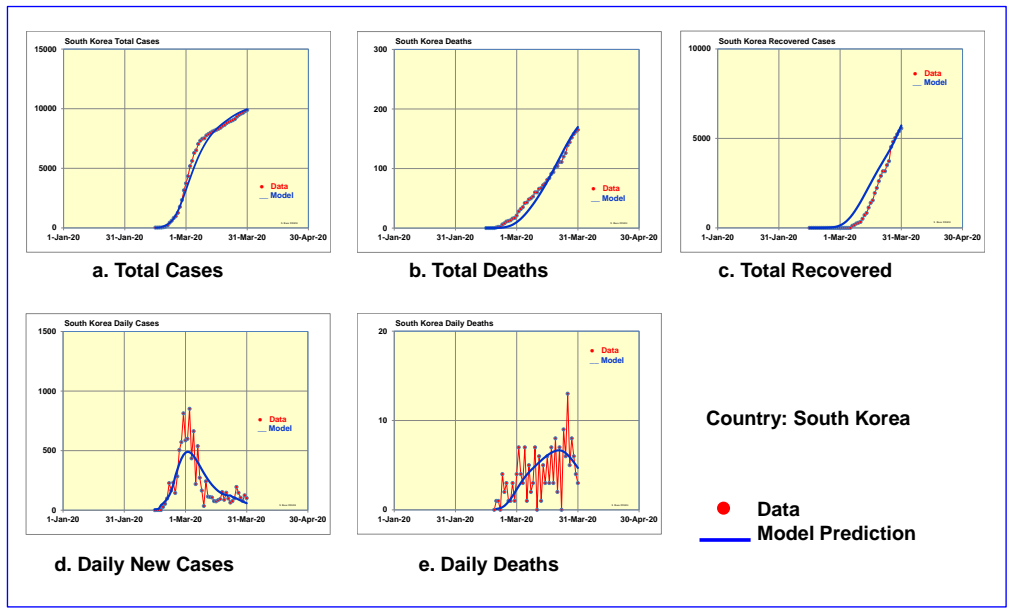

Figure 3. Data and model predictions for Covid-19 cases in South Korea

Figure 4 shows the comparison between the model prediction and the actual data for Iran. The daily case data for Iran has been available in $[5,6,8]$ since Feb 19. There were 2 total cases and equal number of deaths on Feb 19. The model fitted the Iran Covid-19 case data with $\mathrm{T}_{0}=0.31$, Feb 20 start date for transmission factor decrease and a transmission decay rate of $1 / \mathrm{G}$, where $\mathrm{G}=$ 15. For Iran with higher number of casualties than South Korea, the transmission rate decay factor, 
$\mathrm{G}$ is higher (15 as compared to 6). Figure 6 shows the model predicting (solid curve in figure 4(d)) the number of daily new cases to level off at the end of March 2020 timeframe.

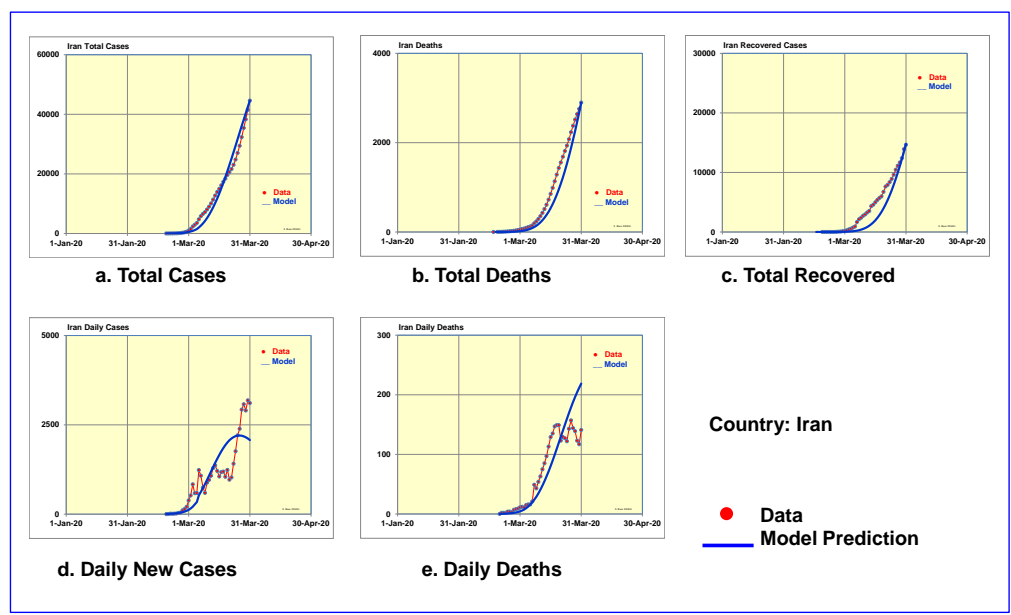

Figure 4. Data and model predictions for Covid-19 cases in Iran

Figure 5 shows the comparison between the Covid-19 case data and the model prediction for Italy. The first reported Covid-19 case in Italy was on Feb 15, at nearly the same time as South Korea and Iran. There were 3 total cases on Feb 19 and the first death was on Feb 21. The model fitted the Italy Coronavirus case data with $\mathrm{T}_{0}=0.38$, Feb 16 start date for transmission factor decrease and a transmission decay rate of $1 / \mathrm{G}$, where $\mathrm{G}=16$. Comparing case data for Italy and South Korea, we find that the model parameter, $\mathrm{G}$ is 16 for Italy as compared to $\mathrm{G}=6$ for South Korea. Transmission containment actions were swifter in South Korea. As shown in figure 7, the model predicts the number of daily new cases reaching a maximum at the end of March 2020 timeframe (solid curve in figure 5(d)).
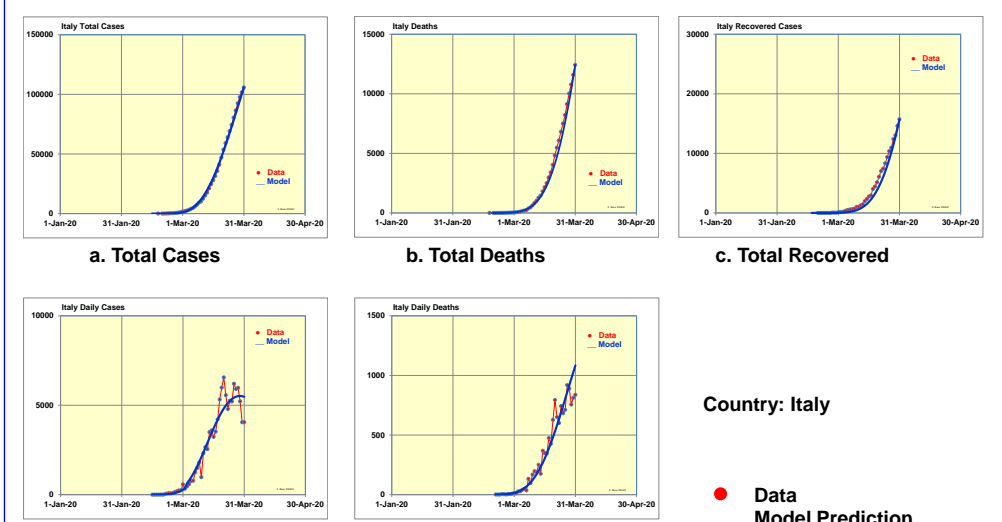

d. Daily New Cases

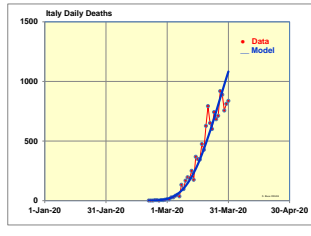

Country: Italy

e. Daily Deaths

- Data

Model Prediction

Figure 5. Data and model predictions for Covid-19 cases in Italy

Figure 6 shows the comparison of the actual data and the model predictions for USA. The daily case data for USA was obtained from references [5-8]. The first reported Covid-19 case in the USA 
was on January 22, 2020. The model fitted the USA Coronavirus case data with minimum error using the following parameters: $\mathrm{T}_{0}=0.22$, Mar 13 start date for transmission factor decrease and a transmission decay rate of $1 / \mathrm{G}$, where $\mathrm{G}=9$. In the USA different measures were taken in different states with population migration among states, the data on which is not fully available at this time. In future it will be possible to extend this mathematical model to the Coronavirus cases in each state separately and then combining to generate a composite model for the country as a whole. The values of the parameters which fit the USA case data in this paper in figure 6 may be considered average values in the country.

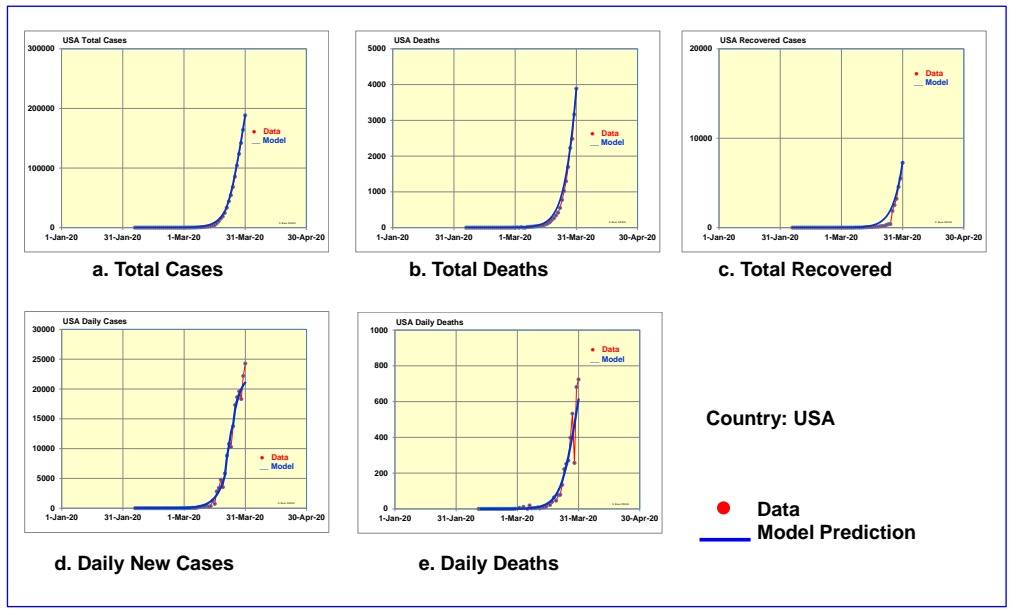

Figure 6. Data and model predictions for Covid-19 cases in USA

\section{Predictive capability}

One of the uses of this model is to be able to predict the course of the outbreak so that corrective actions may be taken to minimize casualties.

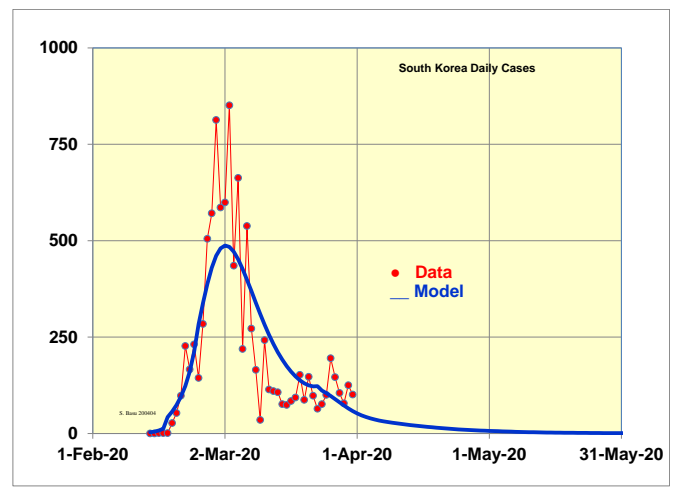

a. Projected Daily Cases

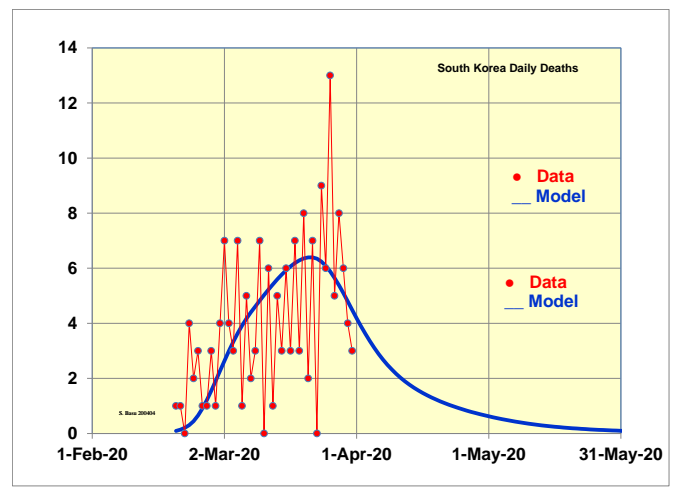

b. Projected Daily Deaths

Figure 7. Predicted daily cases and deaths with the Covid-19 data from South Korea for the next two months

Figure 7 shows the predictions for Covid-19 related daily cases and daily deaths in South Korea for two months (Apr 1 - May 31, 2020) beyond the date range of the used data set. The model is based on the actual case data until Mar $31^{\text {st }}$. Without any change in the model parameters in the 
next two months, the model predicts 741 additional cases and 64 additional deaths. A few comments are in order. The conditions within a country vary daily due to large number of factors which make the model parameters change unpredictably with time. The model predictions are to be considered best estimates with some amount of implied uncertainty. The predictions are only valid for a short period of time in the future. Also for a country such as South Korea, in which the peak of the outbreak has passed, the model should give better predictions than for a country such as India in which the daily number of cases is rising.

One utility for this predictive model is one can take corrective action by knowing what to expect in two weeks from the present by using the model and then adjusting containment strategies by comparing with the actual data.

\section{Sensitivity analysis and what if exercise}

Another use of the model is to be able to carry out sensitivity analysis to see the effects of preventive measures on the outcome. One of the best examples of Covid-19 containment is South Korea with only 9887 cases and 165 deaths until Mar 31 $1^{\text {st }}$, 2020. As explained in the results section, the model fitted the South Korea case data with transmission decay rate $1 / G$, where $G=6$ days. Figure 8 shows the model predictions for Covid-19 for four different scenarios $G=6,8,10$ and 12 days in South Korea. It can be seen that taking 2 days longer to reduce the transmission factor by $63 \%(\mathrm{G}=8$ as compared to $\mathrm{G}=6$ ) would have increased the number of deaths by more than a factor of two, from 230 to 541 . By doubling the number of days to slow down (from $G=6$ to $G=12$ ) would have increased the number of cases by 12 times from 10,639 to 134,486 and the number of deaths from 230 to 2,911 .

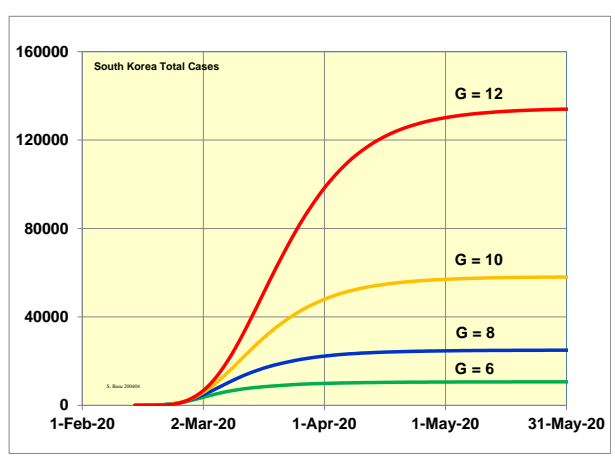

a. Total Cases

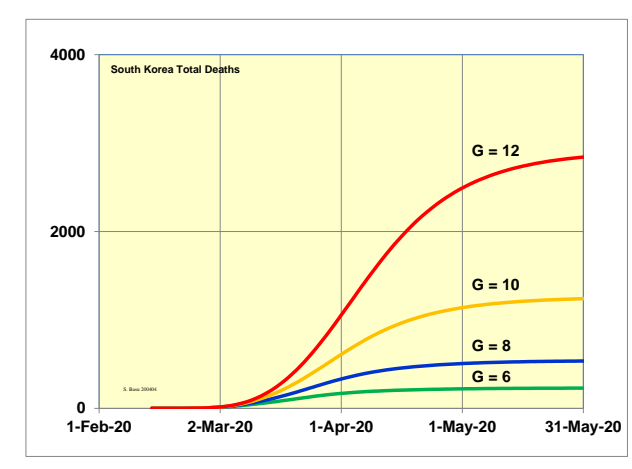

b. Total Deaths

Figure 8. Sensitivity of the total cases and total deaths to the virus transmission reduction factor G using the Covid-19 case data for South Korea

These numbers need to be taken with caution since conditions change daily during a viral outbreak in any country and these have uncertainties associated with them. But the lesson in general is valid which is a great number of deaths in any country can be prevented by taking swift, strict and uniform action throughout the country to prevent person to person viral transmission.

In conclusion, we have developed a general mathematical model that provides insight into the Covid-19 viral outbreak cases in any country. The model fits the data reported until Mar 31, 2020 
in five countries China, South Korea, Iran, Italy and USA with different levels of cases and casualties. The fit is good as seen in figures 2-6. The model is transparent and is based on parameters which are measurable. This allows the model calculations to be updated as the actual data of the parameters become available. One can also use the model parameters as a basis of comparison between the effectiveness of disease containment strategies in different countries. We presented some early results on prediction and sensitivity analyses. In future, we will apply the model to data for other countries. We will also expand the technique to study various cases such as effects of population migration and recurrence of the disease.

\section{Additional Information}

Correspondence and requests for materials should be addressed to the author.

\section{References}

1. Anderson, R.M. and May, R.M., Infectious diseases of humans: dynamics and control (Oxford University Press, Oxford, 1991)

2. Hethcote, H.W. "The mathematics of infectious diseases" Society for Industrial and Applied Mathematics 42, 599-653 (2000)

3. Lin, Q., Zhao, S. et al, "A conceptual model for the coronavirus disease 2019 (COVID-19) outbreak in Wuhan, China and individual reaction and governmental action" International journal of infectious diseases. 93, 211-216 (2020)

4. Kucharski, A.J., Russell, T.W. et al, "Early dynamics of transmission and control of COVID-19: a mathematical modelling study", www.thelancet.com/infection Published online March 11, 2020 https://doi.org/10.1016/S1473-3099(20)30144-4

5. World Health Organization website https://www.who.int/emergencies/diseases/novel-coronavirus-2019

6. World Health Organization website https://www.who.int/docs/default-source/coronaviruse/who-chinajoint-mission-on-covid-19-final-report.pdf

7. U.S. Center for Disease Control and Prevention website https://www.cdc.gov/coronavirus/2019ncov/index.html

8. https://www.worldometers.info/coronavirus/

9. Nishiura, H., Miyama, T. et al, "Estimation of the asymptomatic ratio of novel coronavirus infections (COVID-19)”, to appear in International journal of infectious diseases (2020). 


\section{APPENDIX}
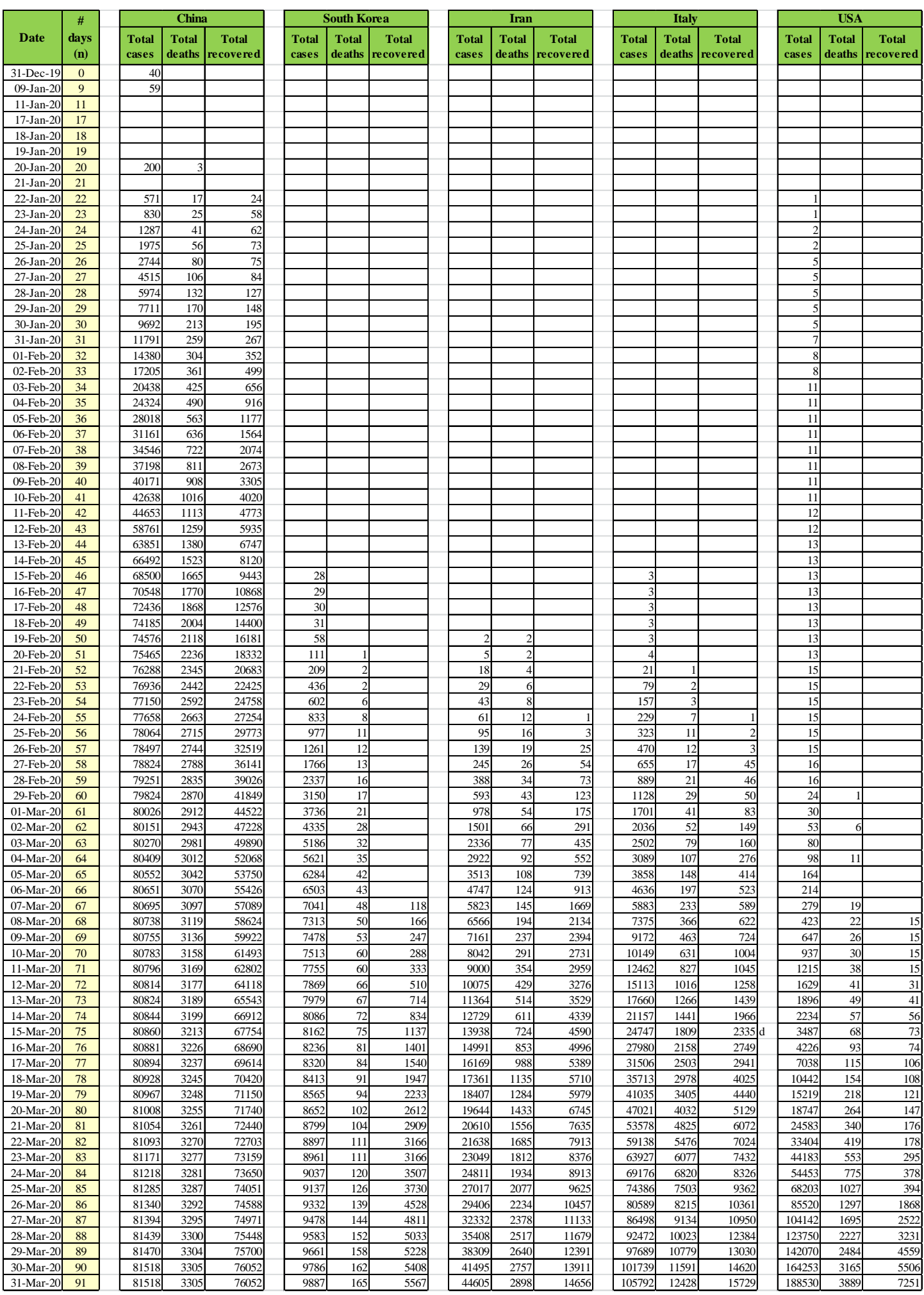

Table I. Covid-19 case data for China, South Korea, Iran, Italy and USA (Jan 1- Mar 31, 2020) 